\title{
Systemic Intravenous Thrombolysis for Massive Pulmonary Embolism after Intracranial Surgery for Traumatic Head Injury: When Absolute Becomes Relative!
}

\author{
Varun Jain ${ }^{1}$ Harsh Sapra ${ }^{2}$ Deepak Bhangale ${ }^{3}$ \\ 1Department of Neuroanaesthesiology and Critical Care, All India \\ Institute of Medical Sciences, New Delhi, India \\ ${ }^{2}$ Department of Neuroanaesthesiology and Critical Care, \\ Medanta-The Medicity, Gurugram, Haryana, India \\ ${ }^{3}$ Department of Neurosurgery, Medanta-The Medicity, Gurugram, \\ Haryana, India
}

J Neuroanaesthesiol Crit Care 2018;5:108-110.

\begin{abstract}
Keywords

- massive pulmonary embolism

- alteplase

- traumatic head injury

- thrombolysis
\end{abstract}

\begin{abstract}
Address for correspondence Varun Jain, MBBS, MD, DNB, DM, Department of Neuroanaesthesiology and Critical Care, All India Institute of Medical Sciences, New Delhi 110029, India (e-mail: varunj2202@gmail.com).
\end{abstract}

\section{Introduction}

Trauma patients have a high risk of venous thromboembolism, with the incidence of deep vein thrombosis (DVT) reaching as high as $58 \%$ in case of traumatic head injury. ${ }^{1}$ Pulmonary embolism (PE) is a known complication in neurocritical care intensive care unit (ICU) where patients have low Glasgow coma scale (GCS) score, with limited mobility and hesitation by clinicians in initiating pharmacoprophylaxis to prevent DVT. Massive PE is a life-threatening situation with intravenous tissue plasminogen activator (tPA) thrombolysis or urgent mechanical thrombectomy as only options. Mortality rate from massive PE varies from 50 to $100 \%{ }^{2}$ When appropriate expertise and resources are available, catheter-guided retrieval of thrombus is suggested over no treatment, when high risk of bleeding exists and the patient has acute PE with hypotension. ${ }^{3}$

\section{Case Description}

A 40-year-old man, road traffic accident victim, with no known/existing comorbidities, was brought to the hospital with an arrival GCS score of E2M4Vt. Noncontrast computed tomography (CT) of the head was suggestive of multiple frontal bone fractures, anterior cranial fossa fractures, multiple hemorrhagic contusions, subarachnoid hemorrhage (SAH), and pneumocephalus in bilateral frontal and basifrontal lobes ( - Fig. $\mathbf{1}$ ). CT of the whole spine was normal. The patient had multiple rib fractures but no long bone fractures. Complete blood count, liver and renal function tests, and two-dimensional (2D) echo were normal with ejection fraction of $65 \%$. Sequential compression stockings were applied as per the ICU protocol to prevent DVT.

On day 1, the patient underwent bifrontal craniotomy, right frontal contusectomy, anterior cranial fossa repair received

February 21, 2018

accepted

April 21, 2018

published online

May 12, 2018
DOI https://doi.org/

10.1055/s-0038-1654752.

ISSN 2348-0548.
Copyright $\odot 2018$ Indian Society of Neuroanaesthesiology and Critical Care

\section{License terms}

(요 (1) $\odot \circledast$ 

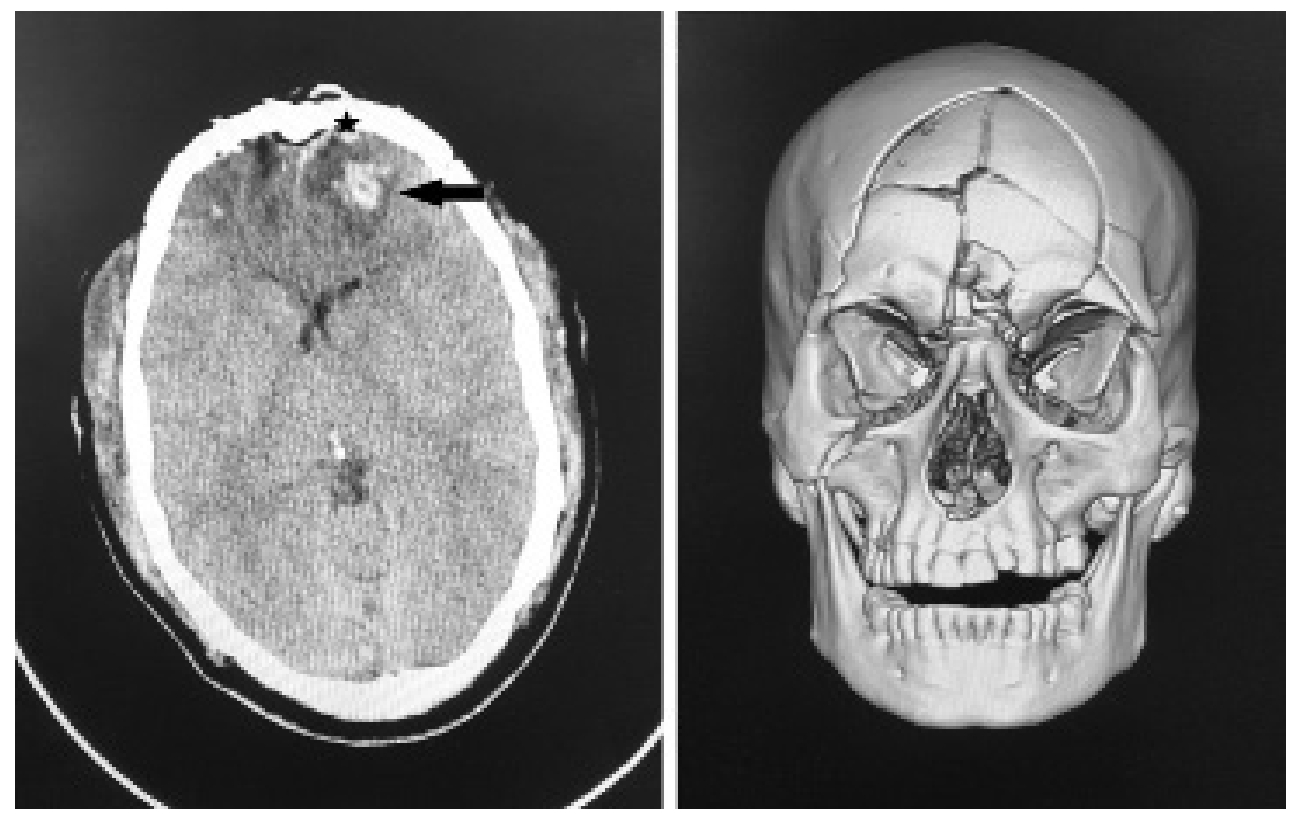

Fig. 1 The non-contrast computed tomography (NCCT) of the head with 3D reconstruction shows multiple frontal bone fractures, anterior cranial fossa fractures, multiple hemorrhagic contusions bilateral frontal and basifrontal lobes (black arrow). SAH in bilateral frontoparietal lobes. Intraventricular hemorrhage present. Pneumocephalus (black star) in bilateral frontal and temporal lobes. 3D, three-dimensional; SAH, subarachnoid hemorrhage.

(by neurosurgical team), and open reduction and internal fixation of the frontal, zygomatic and maxillary bones (by plastic surgery team). On day 2 , he underwent redo bilateral frontal craniotomy for hematoma evacuation. From days 3 to 12 , he had intermittent fever spikes (for which he underwent cerebrospinal fluid [CSF] analysis by lumbar puncture on days 5 and 11 , and meningitis was ruled out) and one episode of seizures on day 5. GCS score during this time fluctuated between E2VtM3 and E2VtM2. Enoxaparin at $40 \mathrm{mg}$ once daily for thromboembolism prophylaxis was started on day 12 . By day 15 , the patient was successfully weaned off ventilator to T-piece for 48 hours. Fever spikes (up to $38.9^{\circ} \mathrm{C}$ ) intermittently continued.

On day 16 morning, the patient had fever $\left(39^{\circ} \mathrm{C}\right)$, was tachypneic (respiratory rate $\approx 25$ breaths/min) and was started on continuous positive airway pressure (CPAP) support. Systolic blood pressure (SBP) was low $(\approx 90-100 \mathrm{~mm} \mathrm{Hg}$ ) that responded to fluid boluses. At this time, 2D echo was normal with no obvious lower limb swellings. Serum procalcitonin was measured (suspecting a new-onset infection), which came $1.0 \mathrm{ng} / \mathrm{mL}$ (similar to the value 4 days back). In the evening, however, the patient suddenly became severely tachypneic (respiratory rate $\approx 35-40$ breaths $/ \mathrm{min}$ ), with desaturation $\left(\mathrm{SpO}_{2}\right.$ up to $92 \%$ on $\left.\mathrm{FiO}_{2} 50 \%\right)$ and needed progressively higher ventilatory and inotropic support. At this time, 2D echo done was suggestive of severe pulmonary hypertension, dilated right atrium and ventricle, and right ventricular ejection fraction (RVEF) 35\%. CT pulmonary angiography was suggestive of bilateral pulmonary artery thrombosis in the main, ascending, descending, and subsegmental branches (-Fig. 2). CT venography showed partial filling defect of the left common femoral, popliteal, and tibial veins and right superficial femoral and popliteal vein.
As the patient's hemodynamics became progressively unstable (SBP $\approx 60 \mathrm{~mm} \mathrm{Hg}$ on triple inotropes), he was considered unsuitable for mechanical thrombectomy or catheter guided thrombolysis. With no better alternative other than intravenous thrombolysis, after consulting and explaining the eventuality to family including high risk of intracranial bleed, the patient was started on alteplase $100 \mathrm{mg}$ over 6 hours followed by heparin infusion for 24 hours. His blood pressure (BP) gradually improved over 6 hours, and he was off inotropes in next 24 hours. GCS was E1VtM1 for next 3 days but then gradually improved. The patient underwent vena cava filter (IVC) filter placement on day 17 and was later started on rivaroxaban. He was shifted to the ward from ICU on day 35 with GCS of E3VtM5 where his GCS improved to E3VtM6 by day 60 .

\section{Discussion}

The authors believe that the use of alteplase for thrombolysis in case of traumatic brain injury with recent decompressive craniectomy for massive PE has not been described previously in the literature. A significant head trauma in previous 3 months, history of intracranial hemorrhage, and recent intracranial surgery are (absolute) exclusion criteria for alteplase administration after acute stroke ${ }^{4,5}$ because of the risk of worsening intracranial hemorrhage. Therefore, massive PE in neurocritical care unit becomes a challenging situation.

Heparinization followed by warfarinization for PE has been done successfully previously in case of a traumatic brain injury with nephrotic syndrome by Akimoto et al. ${ }^{6}$ They noticed slight increase in the hemorrhagic contusions with this therapy. Bottinor et al used tPA in case of a massive PE to save life of a 60 -year-old who had suffered from hemorrhagic 

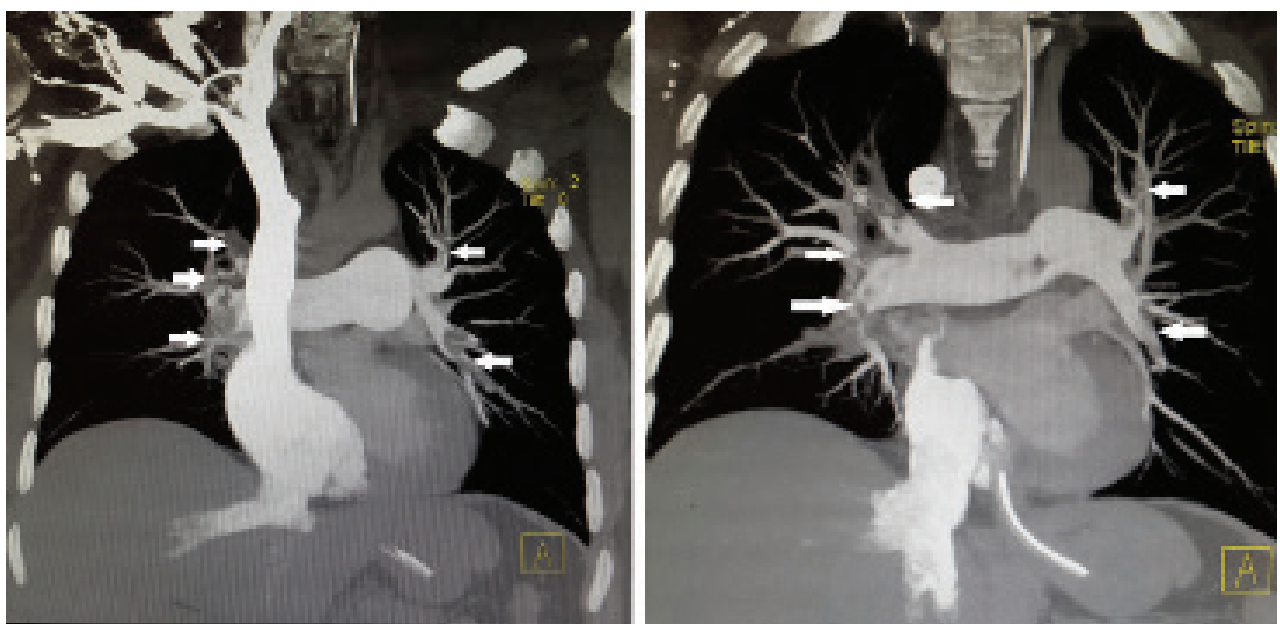

Fig. 2 The CT pulmonary angiography showing filling defect (white arrows) of right and left main pulmonary artery, its ascending, descending, and subsegmental branches. CT, computed tomography.

stroke 8 weeks prior. ${ }^{2}$ Their daring trial achieved muchneeded appreciation, so we should not follow the guidelines blindly but must also make clinical judgments as per the need of situation. ${ }^{7}$ Similarly, thrombolytic bolus was successful in saving a patient's life following intracranial surgery for glioblastoma multiforme who suffered from cardiac arrest while being prepared for the mechanical thrombolysis via ultrasonography for pulmonary embolus dissolution. ${ }^{8}$

With this case report, we wish to highlight that although intravenous thrombolysis is contraindicated in cases of recent head injury and intracranial bleed, even this absolute contraindication should be considered relative in case of an eventually dying patient. ${ }^{5,9}$ We were left with no option to save the patient's life but to try intravenous thrombolysis. ${ }^{3}$ In this case, our patient fortunately recovered from PE with no complication of thrombolysis. Having said that, we acknowledge the wait in starting enoxaparin early and diagnosing PE earlier where we could have stepped up our antithrombotic measures. Therefore, one must be more vigilant in suspecting PE in neurosurgical ICU where antithrombotic measures are usually delayed or underdosed. ${ }^{1,10}$ Fever, a consistent sign in our patient was probably due to DVT, and its association carries worse outcome. ${ }^{11}$

\section{Conclusion}

We thus recommend that every effort must be made for prevention and early diagnosis of DVT and PE in neurocritical care unit, as role of thrombolysis in case of massive PE is limited. If, however, a dire situation arises to save a patient, all the absolute contraindications for thrombolysis become relative (as recent intracranial surgery and cerebral contusions in this case). The clinician must not be hesitant in counseling the family and taking the best possible course at that moment.
2 Bottinor W, Turlington J, Raza S, et al. Life-saving systemic thrombolysis in a patient with massive pulmonary embolism and a recent hemorrhagic cerebrovascular accident. Tex Heart Inst J 2014;41(2):174-176

3 Kearon C, Akl EA, Ornelas J, et al. Antithrombotic Therapy for VTE Disease: CHEST Guideline and Expert Panel Report. Chest 2016;149(2):315-352

4 Jauch EC, Saver JL, Adams HP Jr, et al; American Heart Association Stroke Council; Council on Cardiovascular Nursing; Council on Peripheral Vascular Disease; Council on Clinical Cardiology. Guidelines for the early management of patients with acute ischemic stroke: a guideline for healthcare professionals from the American Heart Association/American Stroke Association. Stroke 2013;44(3):870-947

5 Vedantham S, Piazza G, Sista AK, Goldenberg NA. Guidance for the use of thrombolytic therapy for the treatment of venous thromboembolism. J Thromb Thrombolysis 2016;41(1):68-80

6 Akimoto T, Yamazaki T, Kusano E, Nagata D. Therapeutic dilemmas regarding anticoagulation: an experience in a patient with nephrotic syndrome, pulmonary embolism, and traumatic brain injury. Clin Med Insights Case Rep 2016;9:103-107

7 Fred HL. Is it ever wise to disregard absolute practice guidelines? Absolutely. Tex Heart Inst J 2014;41(2):177-178

8 Bayram B, Oray NÇ, Korkmaz E, Erdost HA, Gokmen N. Massive pulmonary embolism and cardiac arrest; thrombolytic therapy in a patient with recent intracranial surgery and glioblastoma multiforme. Am J Emerg Med 2014;32(11): 1441.e1-1441.e3

9 Mercer RM, Bowen JST, Armstrong RJ. All contraindications to thrombolysis for life-threatening pulmonary embolus should be considered relative. BMJ Case Rep 2013;2013: bcr2013009724

10 Nyquist P, Bautista C, Jichici D, et al. Prophylaxis of venous thrombosis in neurocritical care patients: an evidence-based guideline: a statement for healthcare professionals from the Neurocritical Care Society. Neurocrit Care 2016;24(1):47-60

11 Barba R, Di Micco P, Blanco-Molina A, et al. Fever and deep venous thrombosis. Findings from the RIETE registry. J Thromb Thrombolysis 2011;32(3):288-292

\section{References}

1 Tracy BM, Dunne JR, O’Neal CM, Clayton E. Venous thromboembolism prophylaxis in neurosurgical trauma patients. J Surg Res 2016;205(1):221-227 